\title{
Epigenetic Mechanisms in Psychiatry
}

\author{
Neuropsychopharmacology Reviews (2013) 38, I-2; doi:I0.1038/npp.20 I 2. 185
}

$\mathrm{T}$ his sixth issue of Neuropsychopharmacology Reviews is focused on epi- (Greek for 'over' or 'above') genetics, which, in its broadest definition, concerns the regulation of chromatin structure and function (including but not limited to gene expression) in dividing and nondividing cells. Many epigenetic markings such as DNA methylation, post-translational histone modifications, and histone variants, in concert, define three-dimensional genome architectures inside the nucleus and are thought to serve as a 'molecular bridge' by which myriads of external (environmental) and internal factors mold and shape the nascent genetic material throughout the brain and peripheral tissues over the entire lifespan of an organism. Over the past $\sim 15$ years, epigenetic approaches have moved center stage in many areas of translational and experimental medicine, and neurology and psychiatry are no exception to this trend. From the viewpoint of basic and clinical neuroscience, there are several important emerging insights that fuel interest and excitement for the field of 'neuroepigenetics': (i) many epigenetic markings remain 'plastic' throughout all periods of brain development and aging, with ongoing and highly dynamic regulation occurring in neurons and other differentiated cell types; (ii) some chromatin-modifying drugs-compounds with inhibitory activity directed against histone deacetylases are a well known example-exert profound behavioral effects in animal models of learning and memory, reward- and motivation-related paradigms, and neurodegenerative disorders, pointing to the therapeutic potential of epigeneticdirected approaches for a range of neurological and psychiatric disease; (iii) monogenetic disorders associated with widespread chromatin defects in brain cover a much wider continuum of illness than previously thought, ranging from neurodevelopmental defects in early life to adult onset psychosis and dementia; and (iv) the still largely unexplored concept of trans-generational epigenetic inheritance whereby certain consequences of behavioral experience during life might conceivably be passed onto subsequent generations. For example, early evidence suggests that environmental conditions affecting nutrition, as well as physical and emotional health of a parent, might have lasting consequences for metabolism and affective states in offspring, possibly by affecting promoter- or enhancer-related DNA methylation of relevant genes.

These key concepts, as they pertain to epigenetic regulation and dysregulation in the nervous system, are well represented by the 15 reviews published in this issue. The first set of papers includes an in-depth introduction to some of the key molecular mechanisms in the field of epigenetics, as well as a synopsis of their role in normal and diseased brain. Maze et al (2012) provide a highly informative overview of chromatin and nucleosome remodeling and, in addition, highlight recent insights into the role of histone modifications and histone variants in the context of normal and abnormal development and plasticity (a nucleosome is viewed as the fundamental unit of chromatin, or $146 \mathrm{bp}$ of DNA wrapped around an octamer of histone proteins; chromatin fibers are essentially arrays of nucleosomes connected by linker DNA and linker histones). Next, Moore et al (2012) provide a concise introduction to genomic distribution patterns and developmental regulation of DNA cytosine methylation and of DNA methyltransferases and demethylase pathways-which were discovered only recently. The authors emphasize the dynamic regulation of DNA cytosine methylation (and of a related mark, cytosine hydroxy-methylation), which extends beyond the developmental period, is maintained in adult brain, and is potentially altered in diseases such as schizophrenia. Chess (2012) then discusses the extremely interesting, but still poorly understood, phenomenon of monoallelic expression, by which perhaps more than $5 \%$ of transcripts show an unequal expression in a diploid genome-including the extreme case of complete shutdown of one of the two alleles-despite being genetically identical and residing in the same nucleus.

Following these introductory overview articles, several papers, each focused on a specific spectrum of psychiatric symptoms and a selected set of epigenetic regulators (mostly acetylation and methylation), review recent preclinical work, which demonstrates how findings from small laboratory animals open up new views on the neurobiology and potential treatment for cognitive defects and memory impairment (Peixoto and Abel, 2012), learned fear and post-traumatic stress disorder (Zovkic and Sweatt, 2012), depression (Sun et al, 2012), cocaine addiction (Rogge and Wood, 2012), and neurodevelopmental syndromes (Na et al, 2012). It is our hope and expectation that these papers will stimulate interest in exploring epigenetic drug targets for the treatment of neuropsychiatric disorders, as well as excite the younger generation of readers about basic and translational research in psychiatry.

Because many epigenetic markings show excellent preservation in human postmortem brain, a significant body of literature already exists on developmental or diseaseassociated changes in DNA methylation and histone modifications, including in areas such as the prefrontal cortex that 
lack a clear anatomical correlate in rodent brain (Houston et al, 2012). The review by Grayson and Guidotti (2012) describes how detailed analyses of DNA methylation at GABAergic gene promoters (which are frequently dysregulated in patients on the psychosis spectrum) in human and mouse brain lead them and other groups to the conclusion that chromatin alterations contribute to transcriptional dysregulation in cortical inhibitory interneurons in schizophrenia and bipolar disorder. Similarly, Zhang et al (2012) provide an in-depth analysis on promoter architectures of stress-related glucocorticoid receptor genes in human and rodent hippocampus, and illustrate how parental care or adverse environments (including maltreatment) in early childhood leaves a lasting imprint on epigenetic regulation of steroid receptors and other genes (including the GABA synthetic enzyme GAD67). The resulting changes could then lead to an altered stress responses (rat, mouse) or increased risk for depression and suicide (human).

Not all of the short- or long-term changes in neuronal (or glial) RNA and protein levels in response to environmental stimuli are due to epigenetic regulation of the corresponding gene promoters, with plenty of other regulatory mechanisms involved. One such mechanism that has recently moved to the forefront of the field is micro- and other small RNA-mediated post-transcriptional control of messenger RNA stability and protein translation. These important topics are represented by three papers in this issue. O'Carroll and Schaefer (2012) offer a timely overview on the principles of microRNAmediated regulation of the brain's transcriptome, including specific examples of microRNA-target RNA interactions implicated in normal brain development or in various neuropsychiatric illnesses. Jonkman and Kenny (2012) review how cocaine exposure in the brain's reward circuitry affects microRNA-mediated modulation of key transcriptional activators and repressors such as cAMP response element binding protein and methyl-CpG binding protein 2 , and how these molecular adaptations in turn influence addictionrelated behaviors in animal models. Hwang et al (2012) nicely dissect the molecular pathophysiology of neuronal cell death associated with ischemic stroke and epilepsy, and highlight a complex, multilayered process that involves the RE-1 silencing and transcription factor (REST), repressive chromatin remodeling at the site of target genes, and multiple microRNAs (including REST-sensitive microRNAs).

Although the aforementioned reviews are mostly focused on environment-, drug-, or disease-mediated mechanisms affecting epigenetic-mediated transcriptional and posttranscriptional regulation in postnatal and adult brain, Bohacek and Mansuy (2012) discuss the extremely interesting (and at least from the viewpoint of brain disorders), relatively novel and provocative concept of epigenetic inheritance. Evidence from human and animal studies not only point to lasting effects of early prenatal stress on brain function and behavior in adult offspring, but there is intriguing evidence that not only environmental toxins, but also diet and even environmental enrichment, may mediate phenotypic variations in the offspring via germline transmission, by mechanisms that again may involve DNA methylation/histone modifications or small RNAs.

The rapid and exciting pace of new discoveries in the field of neuroepigenetics also radiate through the various 'hot topic' articles that conclude the 2013 issue of Neuropsychopharmacology Reviews. We, in our function as volume editors, had the special privilege to work with an extremely competent and skilled group of colleagues, including Gwenn Smith, Peter Kalivas, William Carlezon, and James Meador-Woodruff. We are also grateful for outstanding editorial assistance first by Diane Drexler and more recently by Natalie Marler.

\section{ACKNOWLEDGEMENTS}

Work in SA's laboratory is supported by grants from the NIH, DOD/DARPA, and Autism Speaks; work in EJN's laboratory is supported by grants from NIH.

\section{DISCLOSURE}

The authors declare no conflict of interest.

\section{REFERENCES}

Bohacek J, Mansuy IM (2012). Epigenetic inheritance of disease and disease risk. Neuropsychopharmacol 37: 220-236.

Chess A (2012). Random and non-random monoallelic expression. Neuropsychopharmacol 37: 55-61.

Grayson DR, Guidotti A (2012). The dynamics of DNA methylation in Schizophrenia (SZ) and related psychiatric disorders. Neuropsychopharmacol 37: 138-166.

Houston I, Peter CJ, Mitchell A, Straubhaar J, Rogaev E, Akbarian S (2012). Epigenetics in the human brain. Neuropsychopharmacol 37: 183-197.

Hwang JY, Aromolaran KA, Zukin RS (2012). Epigenetic mechanisms in stroke and epilepsy. Neuropsychopharmacol 37: 167-182.

Jonkman S, Kenny PJ (2012). Molecular, cellular and structural mechanisms of cocaine addiction: a key role for microRNAs. Neuropsychopharmacol 37: 198-211.

Maze I, Noh KM, Allis CD (2012). Histone regulation in the CNS: basic principles of epigenetic plasticity. Neuropsychopharmacol 37: 3-22.

Moore LD, Le T, Fan G (2012). DNA methylation and its basic function. Neuropsychopharmacol 37: 22-38.

$\mathrm{Na}$ ES, Nelson ED, Kavalali ET, Monteggia LM (2012). The impact of MeCP2 loss or gain of function on synaptic plasticity. Neuropsychopharmacol 37: 212-219.

O'Carroll D, Schaefer A (2012). General principals of miRNA biogenesis and regulation in the brain. Neuropsychopharmacol 37: 39-54.

Peixoto L, Abel T (2012). The role of histone acetylation in memory formation and cognitive impairments. Neuropsychopharmacol 37: 62-76.

Rogge G, Wood MA (2012). The role of histone acetylation in cocaine-induced neural plasticity and behavior. Neuropsychopharmacol 37: 94-110.

Sun H, Kennedy PJ, Nestler EJ (2012). Epigenetics of the depressed brain: role of histone acetylation and methylation. Neuropsychopharmacol 37: 124-137.

Zhang TY, Labonté B, Wen XL, Turecki G, Meaney MJ (2012). Epigenetic mechanisms for the early environmental regulation of hippocampal glucocorticoid receptor gene expression in rodents and humans. Neuropsychopharmacol 37: 111-123.

Zovkic I, Sweatt JD (2012). Epigenetic mechanisms in learned fear: implications for PTSD. Neuropsychopharmacol 37: 77-93.

Schahram Akbarian ${ }^{1}$ and Eric J Nestler ${ }^{1}$

${ }^{1}$ Departments of Neuroscience and Psychiatry, Friedman Brain Institute, Mount Sinai School of Medicine, New York, NY, USA E-mail: schahram.akbarian@mssm.edu or eric.nestler@mssm.edu 